\title{
Model-based optimal therapy for high-impact diseases
}

\author{
L. Kovács*, J. Sápi*, T. Ferenci*, P. Szalay**, D.A. Drexler**, Gy. Eigner*, P.I. Sas*, B. Kiss*, I. Harmati**, \\ M. Kozlovszky*, Z. Sápi*** \\ * Óbuda University, Budapest, Hungary \\ \{kovacs.levente, kozlovszky.miklos\}@nik.uni-obuda.hu, \{ferenci.tamas, sapi.johanna\}@phd.uni-obuda.hu, \\ eignergyorgy@googlemail.com,sapi35@gmail.com \\ ** Budapest University of Technology and Economics, Budapest, Hungary \\ \{szalay, drexler, harmati\}@iit.bme.hu \\ *** Semmelweis University, Budapest, Hungary \\ sapi.zoltan.dr@gmail.com
}

\begin{abstract}
The current paper represents a continuation of the conference papers presented at the INES 2011 and 2012 conferences. A brief summary of the newest results in the field of physiological modeling and control are presented focusing on high public health impact diseases: diabetes, tumor and obesity. In the case of diabetes control, preliminary model-free results of the developed robust control framework is presented, while in the case of tumor control we have started to create a new tumor growth model based on clinical experiments. In the case of biostatistical investigation of obesity, new aspects of relationship between overweight/obesity and laboratory results was examined.
\end{abstract}

\section{INTRODUCTION}

Physiological control - a subdiscipline of biomedical engineering [1] - studies and applies identification and control strategies in order to understand and help automated treatment of various diseases or injuries of the human body. In the last period the literature focused more and more on individualized model-based control algorithms, but on the other way an important question has been formulated how to generalize the obtained results. The newly formed Physiological Controls Group of the Óbuda University together with the Control Engineering and Information Technology of the Budapest University of Technology and Economics and the Semmelweis University from Budapest are investigating the mentioned problem.

The article briefly presents the newest results obtained as a direct continuation of the results presented at the INES 2011 and 2012 conferences [2], [3].

Diabetes is named by the World Health Organization (WHO) as the "disease of the future". Statistics of the International Diabetes Federation (IDF) show that the European Region has the highest number and the highest incidence rate of type 1 diabetes (T1DM) in children from any other region worldwide [4].

Sensor-augmented pump therapy - using continuous glucose monitors (CGM) for the subcutaneous measurement of glucose concentration and insulin pumps for the subcutaneous delivery of insulin - seems to improve the glycaemic control compared to traditional therapeutic schemes [5].

The automatic regulation of glucose profile using a controller that is closing the loop between CGM and pump will make the realization of an AP [6]. Different control algorithms have been proposed in the literature [6], and those being able to develop a prototype system presented good results achieving nocturnal glucose regulation [6]. However, during the daytime, due to insulin sensitivity differences, patient variability and also other uncontrolled and not modeled events (like stress, physical activity, sensor failure, meal estimation error, etc.) the current approaches cannot give a robust solution; hence, the AP problem remains a huge challenge.

Cancer diseases are leading causes of death nowadays all around the world. In the EU the total predicted number of cancer deaths in 2013 is 1.314.236 compared with 1.281.694 in 2009 [7]. In Hungary, cancer was the second most common cause of death after cardiovascular diseases in 2012 [8].

There are classical therapies, such as chemotherapy and radiation therapy, but chemical agents and ionizing radiation have effects on certain healthy cells of the patient as well. Treatments which are based on specific molecules that target a signalling pathway in the growth and development of a tumor cell are called targeted molecular therapies (TMTs).

Tumor growth is limited by diffusion distance, thus after a certain size tumor development stops in the absence of oxygen and nutrients. When tumor becomes able to form own blood vessels, it is called angiogenic switch. Thereby, tumor leaves premalignant stage and become malignant tumor, whereupon rapid tumor growth starts and after vascular invasion tumor can give metastasis in other organs [9].

Antiangiogenic therapy [10] is a type of TMTs, which inhibits angiogenesis (forming new blood vessels). Preventing tumor cells from grow and develop, the tumor can be kept in a dormant state, where the cellular proliferation rate balanced by the apoptotic rate, thus the tumor will be unable to grow in size beyond a few millimetres [11].

Obesity [12] has reached epidemic proportions in many countries of the developed world. For instance, since a long time more than the half of the United States' population has overweight or is obese [13]. The situation is not much better in Hungary: according to the latest data from the OECD $34.1 \%$ of the Hungarian adult population has overweight, and $19.5 \%$ is obese [14]. Unfortunately 
the same trends apply for pediatric obesity as well: worldwide [15] and in Hungary [16].

Obesity is in focus of public health because it has been demonstrated to increase all-cause morbidity and mortality [17]; hence, it represents an important public health risk factor. Diseases that have been casually linked to obesity include type 2 diabetes mellitus, several cardiovascular diseases, asthma, etc. [18]. These have been described specifically in children too [19].

Obesity, but even overweight induces certain changes in laboratory parameters. Several changes are more or less direct consequence of the altered homeostatic equilibrium caused by obesity (for example the elevated levels of alanine transaminase and aspartate transaminase [20]). On the other hand, the change in certain laboratory parameters cannot be attributed to a single, well-defined pathophysiological alteration, but indicate a more complex process, sometimes even before the patient has any symptom of manifest obesity (for instance C-reactive protein [21]).

The relationship between overweight/obesity and laboratory results was investigated empirically, based on a cross-sectional clinical observation. To our recent knowledge, there is no Hungarian database of adolescents which contains the necessary anthropometric, demographic and laboratory parameters; we organized a data collection specifically to address this issue.

\section{PRELIMINARY MODEL-FrEE RESULTS OF THE DEVELOPED ROBUST CONTROL FRAMEWORK}

Last year at the INES 2012 conference we have summarized the validation results obtained on our robust control framework regarding a large set of 83 type 1 diabetic patients [3].

Based on the obtained results, we went further and started to investigate the model-free property of the controller: if the originally used modified Sorensen-model [22] is changed to another model, how should the controller react without redesigning it.

We have used the well-known model of Hovorka [23], and created a whole new set of virtual patients. This model represents the core of the in-silico simulator of the University of Cambridge version 2.2 (SimEdu). Using the results, which were collected from the insulin pump centers of the Hungarian Artificial Pancreas Working Group (MAP), these two T1DM models were used to generate the virtual patients. The identification was carried out using the Levenberg-Marquardt algorithm [24] and the Optimization toolbox of MATLAB. The running time has been greatly reduced by providing a fair estimation of the Jacobian matrix without the use of loops.

Based on our preliminary results we found out that hypoglycaemia is still efficiently avoided and hyperglycaemia is reduced more than $75 \%$ to the real datasets if parameter identification and the starting point of the algorithm are well determined. Simulations were started 1-2 days before the start of the identification timeframe in order to minimize problems caused by initial states. However, this requires more apiori information from the patient side.

Fig. 1 presents simulation results of 14 years old girl using insulin pump from three months, and having an active lifestyle and bad compliance. She is managed at Veszprém, Hungary, using an IPro2 glucose sensor.
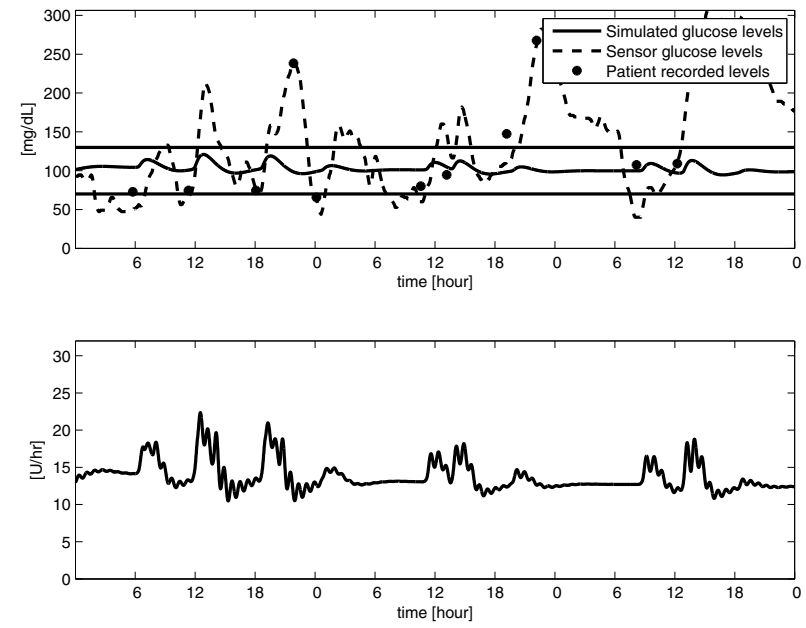

Figure 1. Simulation results on the model of Wilinska et al. [23]. The upper figure presents the comparison of robust controller output (solid line) and sensor real data (dashed line), while the dots represent the real fingertip measurements. The lower figure shows the insulin control input.

It can be seen that blood glucose level could be still stabilized in a better range than the real data of the patient, while hypoglycaemia is efficiently avoided. However, the insulin input is not optimal anymore (compared to the results on the modified Sorensen model), as a bigger continuous value should be used, complemented with other boluses as well.

In the future it is planned to make more model-free tests on the model of [23], but on other models as well.

\section{TUMOR GROWTH MODEL DEVELOPMENT}

\section{A. Control design of existing tumor growth model from the literature}

We have investigated a simplified form of a dynamic model for tumor growth in antiangiogenic therapy introduced in [25]. Continuing our preliminary examinations and results in antiangiogenic tumor growth control [26-29], our aim was to find suboptimal, but close to optimal control solutions, which are cost-effective (angiogenic inhibitors are quite expensive, thus the goal of the control is to minimize the tumor volume using the least possible control signal).

Solving the control problem by linearization [28], we have designed four controllers: state feedback with pole placement, LQ control method, state feedback with pole placement and observer, and LQ control method with observer. Controllers were designed for the linearized model, but were used for the nonlinear model. Three working points, three saturation values and three amount of acceleration were analyzed. Controllers were evaluated by three criteria: (i) the total concentration of the administered inhibitor during the treatment $(\mathrm{mg} / \mathrm{kg})$, (ii) the steady state inhibitor concentration at the end of the treatment $(\mathrm{mg} / \mathrm{kg})$, (iii) the steady state tumor volume at the end of the treatment $\left(\mathrm{mm}^{3}\right)$.

Simulation results were divided into seven groups. Three groups of controls were efficient, but not successful (tumor volume was reduced, but not up to the required volume). Two groups of controls were 
unsuccessful. For almost every controller oscillation was found at the steady state inhibitor concentration, mainly in low working points - these controls form a group as well.

The last group contains the successful control solution with respect of each criterion. LQ controllers had the best results for (i) and (ii) criteria, but also for (iii) criterion these controllers had near-optimal values. In addition, LQ control method was the only one, which could handle the problem of high working points. Therefore, we have investigated weighting matrix $R$ in a wide range $(R=$ $\left.\left[10^{2}, 10^{6}\right]\right)$.

We have found that there are "typical" minimum locations, where each criterion has close to optimal values. Thus we created an optimal set of $R$ values with 8 elements. Since we had 3 examined saturation values, we got 24 optimal $R$ value - saturation value pairs. Considering all three criteria, optimal $R$ value - saturation value pairs, which give the best result, are summarized. We have found, that $R=2000, u_{\max }=25 \mathrm{mg} / \mathrm{kg}$ and $R=$ 4000, $u_{\max }=25 \mathrm{mg} / \mathrm{kg}$ pairs are solutions for each working point, however in medium and high working points the steady state tumor volume is higher than the required value.

Consequently, the control law can be formulated in two phases. In the first phase of the control, $R=2000$, $u_{\text {max }}=25 \mathrm{mg} / \mathrm{kg}$ or $R=4000, u_{\max }=25 \mathrm{mg} / \mathrm{kg}$ pair have to be chosen. In the second phase, this control has to be kept, if the steady state tumor volume is optimal; otherwise a smaller $R$ value has to be chosen, which results an optimal steady state tumor volume also in medium or high working points. Switching ensures that the control passes along optimal points considering all three criteria.

\section{B. Identification of a new tumor growth model}

We have examined tumor growth dynamics in case of C38 colon adenocarcinoma and B16 melanoma with mouse experiments. C38 tumor cells inflict strong hypoxial reaction and have a large relative vascular area [30], which is beneficial if we would like to examine the effect of angiogenic inhibition. B16 melanoma [31] is also a mouse tumor. B16 mice melanoma can be used as a model for human melanoma [32].

The simplest dynamic model is a linear one, thus the response of the system consists of exponentials functions. Antiangiogenic therapy effects on tumor vasculature, the inhibition of vascular system results in the reduction of tumor mass. Since we want to identify the dynamics behind tumor mass growth and vasculature growth, we are seeking for a second order system.

The second order system has two exponential functions in its response, thus parametric identification was carried out by fitting a curve with two exponential functions.

Exponential curve fitting for average in case of $\mathrm{C} 38$ colon adenocarcinoma can be seen in Fig. 2. The response of the system is described by:

$$
y(t)=-0.076 \cdot \exp (0.4239 t)+16.87 \cdot \exp (0.2329 t)
$$

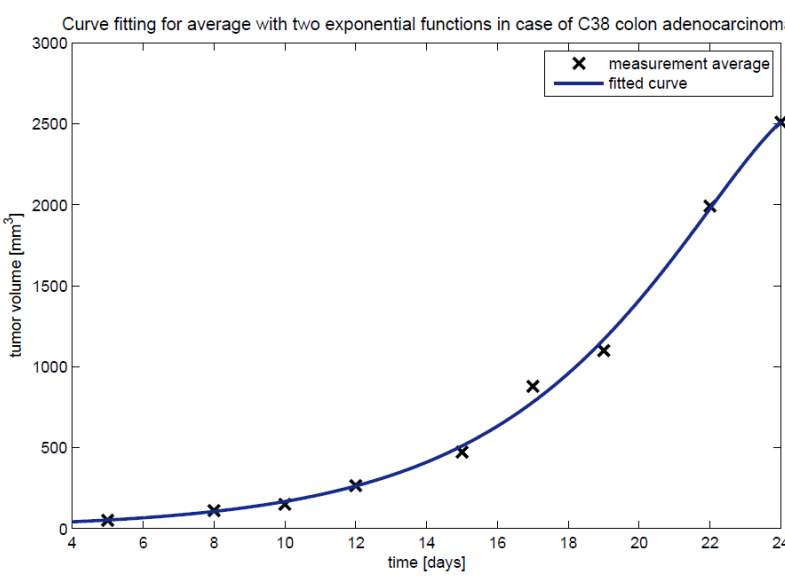

Figure 2. Exponential curve fitting for average in case of $\mathrm{C} 38$ colon adenocarcinoma $(y(t)=-0.076 \cdot \exp (0.4239 t)+16.87 \cdot \exp (0.2329 t))$

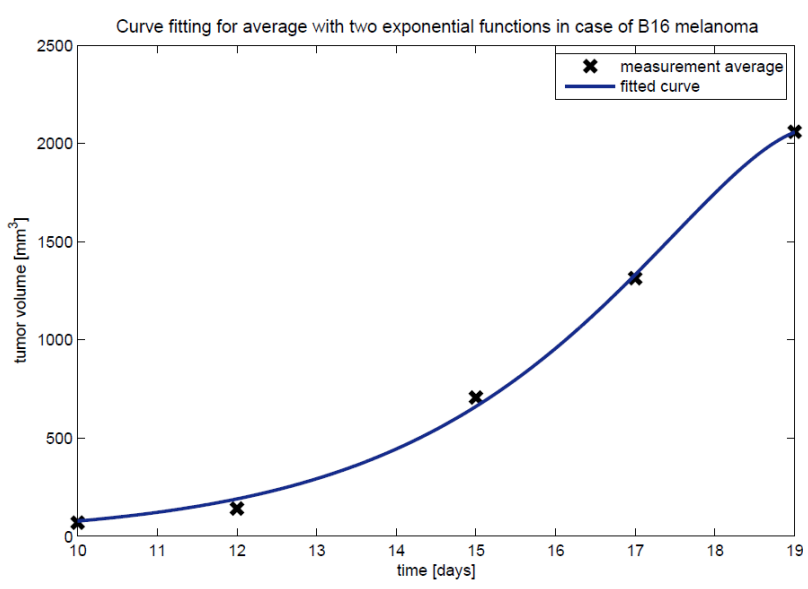

Figure 3. Exponential curve fitting for average in the case of B16 melanoma $(y(t)=-511.6 \cdot \exp (0.54781 t)+512.3 \cdot \exp (0.54775 t))$

The time constants of the identified model are: $T_{1}=$ 2.3589 days, and $T_{2}=4.2938$ days. The coefficients of the exponentials are positive, thus the system is unstable, as it is required from a tumor growth.

Exponential curve fitting for average in case of B16 melanoma can be seen in Fig. 3. The response of the system is described by:

$$
y(t)=-511.6 \cdot \exp (0.54781 t)+512.3 \cdot \exp (0.54775 t)
$$

The time constants of the system are $T_{1}=1.8256$ days and $T_{2}=1.8254$ days. The parametric identification results in almost identical time constants; however, the multiexponential characteristic is important. This simple model results in a plateau-like characteristic at high tumor volume values, without the nonlinear model of the Gompertzian growth [33].

The coefficients of the exponential functions are positive in this case as well, resulting in an unstable system.

In the future we plan to formulate the created model in terms of control engineering, make more validation tests, 
and design control structures similar to ours already presented algorithms.

\section{BiostatisticAl METHOD FOR OBESITY INVESTIGATION}

\section{A. Patient Data}

We have examined 82 healthy volunteer adolescents ( 60 boys and 22 girls) and 101 patients ( 53 boys and 48 girls) treated for obesity without significant comorbodity (Heim Pál Children's Hospital, Budapest). Altogether our sample included $n=183$ adolescents aged between 12 and 18 (113 boys, 70 girls).

For the healthy volunteers, the examination consisted of anthropometric measurements (body height, body mass, hip-, waist- and left arm circumference), body composition analysis, recording of basic demographic and anamnestic data, and the drawing of a fasting blood sample for laboratory examination. The investigated subjects form a convenience sample; hence the results are not necessarily representative. In particular, the obese population was intentionally oversampled in the study design.

The investigation's research program was preauthorized by the Hungarian Regional Bioethical Committee.

\section{B. Statistical Analysis}

We used the well-known Body Mass Index (BMI) to quantify overweight/obesity. The BMI must be considered relatively based on age (and sex). We used $z$ score, which is a standardized BMI (that is: actual BMI minus the reference BMI mean for the age and sex, divided by the standard deviation of the reference for age and sex), with the exception that instead of the actual BMI, an algebraic transform of it is used (which aims to normalize the distribution of BMI, so that $z$-score is interpretable) [34]. According to our knowledge, the only Hungarian growth chart (which is based on the National Longitudinal Child Growth Survey [35]) does not contain the parameters necessary for normalization, so we used the US growth chart of the Centers for Disease Control and Prevention (CDC) [36] to calculate the $z$-scores (or $\left.z_{B M I}\right)$. Sexes were separately analyzed to avoid intractable interactions.

The logic of the investigation is illustrated on Fig. 4 for the white blood cell count of boys. Fig. 4a is the scatterplot of the investigated laboratory parameter and $z_{B M I}$. We used the Spearman (rank)correlation coefficient [37], which measures the strength and direction of not only linear, but monotone connection in general. This way, we could detect potential nonlinear relationships as well. We considered a correlation significant if the $p$ value was less than $0.05 / 33$, that is, the significance level was corrected according to Bonferroni [38] to take the multiple comparisons situation [39] into account.

The next problem was to define how an indicator is measured for a given standardized BMI (for example for
$\left.z_{B M I}=2\right)$. As our data are continuous, the simple calculations are not possible (most likely there is not a single patient who has exactly $z_{B M I}=2$ ). We tried to reconstruct the (two-dimensional) joint distribution (probability density function) of $z_{B M I}$ and the investigated laboratory parameter. For that end, we used kernel density estimation [40] to obtain a (continuous) distribution based on the sample. We used normal kernel, the bandwidth-matrix was estimated with smoothed cross-validation [41]. The estimated two-dimensional joint probability density function is shown (with contour lines) in Fig. 1b.

After this, the procedure was straightforward: from the joint distribution, one can simply obtain the conditional distribution for any $z_{B M I}$ (as condition), and from this conditional distribution, any indicator can be directly calculated (the average, for example, by means of numeric integration). For reasons of interpretability and robustness, we used average and median (as indicators of central tendency) and standard deviation and interquartile range (as indicators of dispersion) [42].

\section{Data Processing}

Data was processed, and statistical analysis was carried out under $\mathrm{R}$ statistical program package (version 14.2). To automate the whole process, we have developed a script which is available at the corresponding author on request.

\section{Results}

These results confirm many theories that are already known from the literature and also raise some new questions.

The level of C-reactive protein is strongly associated with the degree of overweight/obesity, both for boys and for girls. This observation is consistent with the literature: obesity is long considered as a systematic, 'low-grade' inflammation state, even in childhood [43]. Deviations we identified above were else already described, specifically in children too [44].

This is supplemented by the observation that the haemoglobin decreases with the level of obesity (although not significantly after correction), with MCV and $\mathrm{MCH}$ decreasing (significantly for both sexes) - and in fact, chronic inflammation can cause anaemia [45]. The change in MCV and MCH pointed to hypochromic, microcytic anaemia.

Levels of liver enzymes ALT and GGT (but not AST) are positively correlated with the degree of obesity (significant for boys). The most likely explanation for this is the effect of central obesity on liver function which is presumed to be in connection with non-alcoholic fatty liver disease [46], [47].

\section{CONCLUSIONS}

The paper summarized the current research tasks in the field of physiological modeling and control of diseases 
with high public health impact carried out by the recently formed Physiological Controls Group of the Obuda
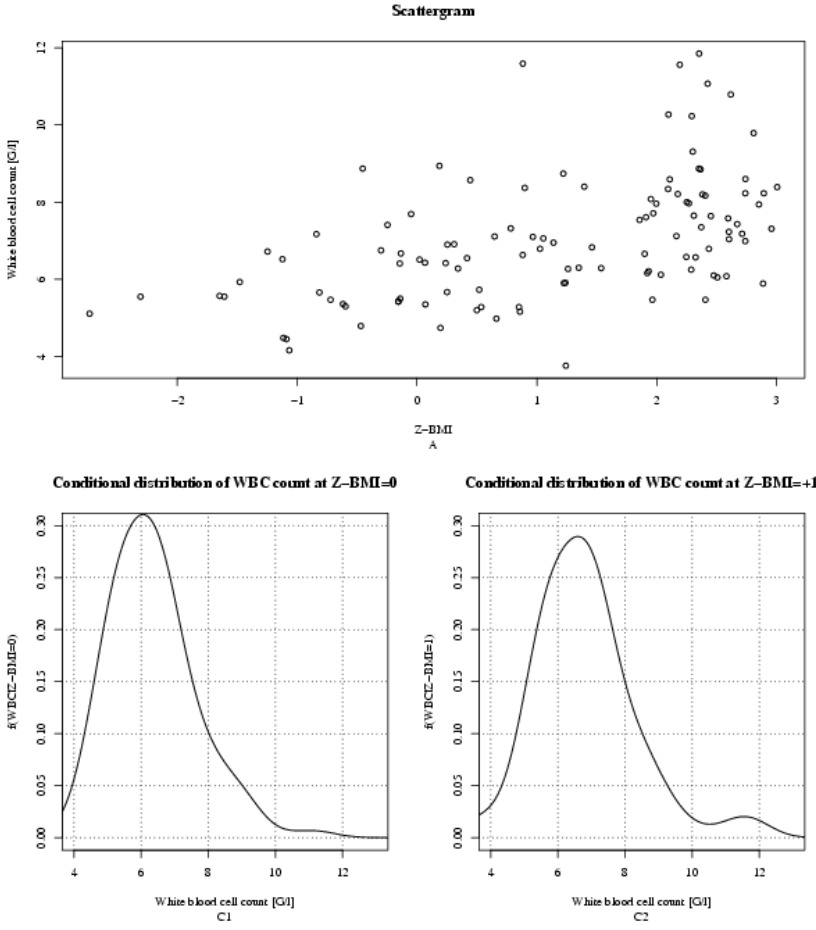

University. Latest results were presented in the field of diabetes, cancer and obesity.
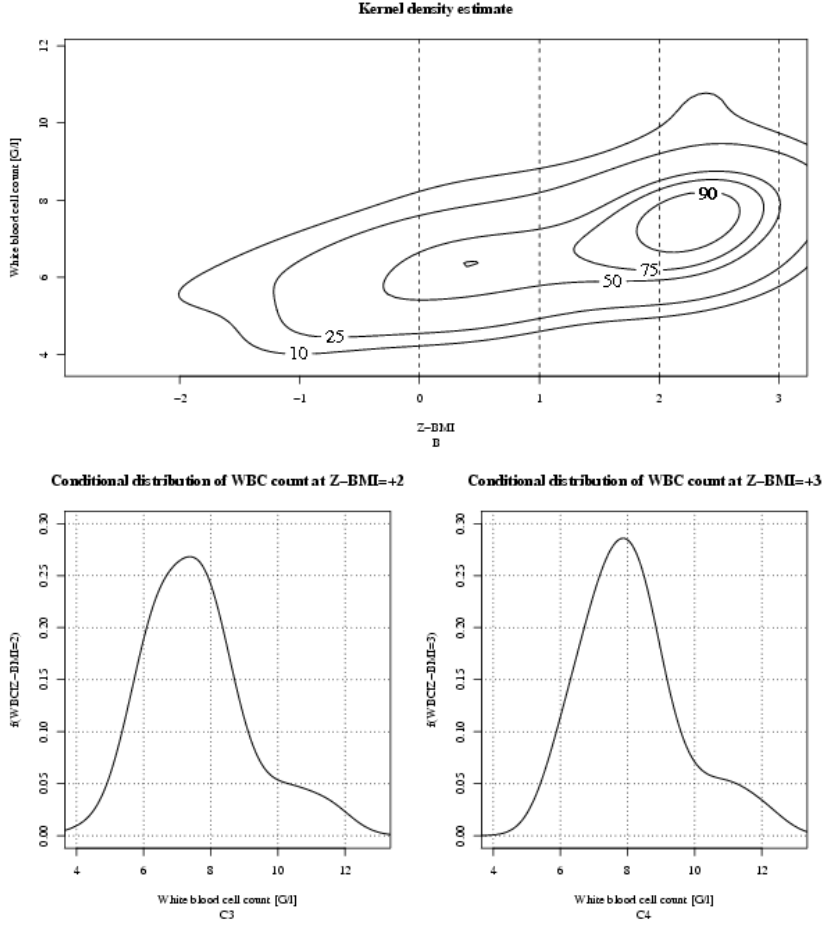

Figure 4. Investigation methodology in case of obese and laboratory parameters through the white blood cells of the boys.

\section{ACKNOWLEDGMENT}

This work was supported by the GOP-1.1.1-11-20120055 project. Levente Kovács is supported by the János Bolyai Research Scholarship of the Hungarian Academy of Sciences.

The authors say special thanks to the Hungarian Artificial Pancreas working group's insulin pump centers for the real data provided to validate the nonlinear modelbased type 1 diabetes robust control algorithm as well as for Prof. Dr. László Barkai, vice president of the Hungarian Diabetes Association for his continuous support.

\section{REFERENCES}

[1] J. Bonzino, The Biomedical Engineering Handbook. CRC in cooperation with IEEE Press, 1995.

[2] L. Kovács, P. Szalay, T. Ferenci, D.A. Drexler, J. Sápi, I. Harmati, and Z. Benyó, "Modeling and Control Strategies of Diseases with High Public Health Impact", in Proc. INES 2011 15th International Conference on Intelligent Engineering Systems 2011, Poprad, Slovakia, pp. 23-28, 2011.

[3] L. Kovács, P. Szalay, T. Ferenci, J. Sápi, P. Sas, D.A. Drexler, I. Harmati, B. Benyó and A. Kovács, "Model-based control algorithms for optimal therapy of high-impact public health diseases", in Proc. INES 2012 - 16th International Conference on Intelligent Engineering Systems 2012, Lisabon, Portugal, pp. 531536, 2012.

[4] http://www.idf.org/diabetesatlas/diabetes-young-globalperspective, 08.04.2013.

[5] R. M. Bergenstal, W. V. Tamborlane, A. Ahmann, J. B. Buse, G. Dailey, N. D. Stephen, C. Joyce, T. Peoples, B. A. Perkins, J. B. Welsh, S. M. Willi and M. A. Wood, "Effectiveness of SensorAugmented Insulin-Pump Therapy in Type 1 Diabetes". New Engl J Med, vol. 363, pp. 311-320, 2010.
[6] C. Cobelli, E. Renard and B. Kovatchev, "Artificial Pancreas: Past, present and future”, Diabetes, vol. 60, no. 11, pp. 2672-2682, 2011.

[7] M. Malvezzi, P. Bertuccio, F. Levi, C. La Vecchia and E. Negri, "European cancer mortality predictions for the year 2013", Ann Oncol, vol. 24(3), pp. 792-800, 2013.

[8] Hungarian mortality statistics of the common causes of death http://www.ksh.hu/docs/hun/xstadat/xstadat_eves/i_wnh001.html, 02.05.2013.

[9] A. Hoeben, B. Landuyt, M. Highley, H. Wildiers, A. T. Van Oosterom and E. A. De Bruijn, "Vascular endothelial growth factor and angiogenesis", Pharmacol Rev., vol. 56, pp. 549-580, 2004.

[10] H. C. Wu, C. T. Huang and D. K. Chang, "Anti-angiogenic therapeutic drugs for treatment of human cancer", $J$ Cancer., vol. 4(2), pp. 37-45, 2008.

[11] J. Pluda, "Tumor-associated angiogenesis: mechanisms, clinical implications, and therapeutic strategies", Semin Oncol., vol. 24(2), pp. 203-218, 1997.

[12] R. Anderson, Obesity: etiology, assessment, treatment, and prevention. Champaign, Human Kinetics Publishers, 2003.

[13] C. Ogden and M. Carroll, Prevalence of Overweight, Obesity, and Extreme Obesity Among Adults: United States, Trends 1960-1962 Through 2007-2008, National Center for Health Statistics; 2010.

[14] OECD Factbook 2011-2012, Economic, Environmental and Social Statistics.

[15] Y. Wang and T. Lobsten, "Worldwide trends in childhood overweight and obesity", Int $J$ of Ped Obes., vol. 1, pp. 11-25, 2006.

[16] M. Antal, Sz. Péter, L. Biró, K. Nagy, A. Regöly-Mérei, Gy. Arató, Cs. Szabó and É. Martos, "Prevalence of Underweight, Overweight and Obesity on the Basis of Body Mass Index and Body Fat Percentage in Hungarian Schoolchildren: Representative Survey in Metropolitan Elementary Schools", Ann Nutr Metab vol. 54, pp. 171-176, 2009.

[17] T. Visscher and J. Seidell, "The public health impact of obesity", Ann Rev Public Health, vol. 22, pp. 355-375, 2001. 
[18] D. Guh, W. Zhang, N. Bansback, Z. Amarsi, L Birmingham and A. Anis, "The incidence of co-morbidities related to obesity and overweight: a systematic review and meta-analysis", BMC Public Health, vol. 9, pp. 88, 2009.

[19] G. Nyberg, U. Ekelund, T. Yucel-Lindberg, T. Modeér and C. Marcus, "Differences in metabolic risk factors between normal weight and overweight children", Int J Pediatr Obes, vol. 6, pp. 244-252, 2011.

[20] C. Ruhl and J. Everhart, "Determinants of the association of overweight with elevated serum alanine aminotransferase activity in the United States", Gastroenterology, vol. 124, pp. 71-79, 2003.

[21] K. L. Ong, A. W. Tso, A. Xu, L. S. C. Law, M. Li, N. M. S. Wat, K. A. Rye, T. H. Lam, B. M. Y. Cheung and K. S. L. Lam, "Evaluation of the combined use of adiponectin and C-reactive protein levels as biomarkers for predicting the deterioration in glycaemia after a median of 5.4 years", Diabetologia, vol. 54, no. 10, pp. 2552-2560, 2011.

[22] R. S. Parker, F. J. Doyle III, J. H. Ward. and N. A. Peppas, "Robust $\mathrm{H}_{\infty}$ Glucose Control in Diabetes Using a Physiological Model", AIChE Journal, vol. 46, no. 12, pp. 2537-2549, 2000.

[23] M. E. Wilinska, L. J. Chassin, C. L. Acerini, J. M. Allen, D. B. Dunger and R. Hovorka, "Simulation environment to evaluate closed-loop insulin delivery systems in type 1 diabetes", $J$ Diabetes Sci Technol, vol. 4, no. 1, pp. 132-144, 2010.

[24] J. Nocedal and S. J. Wright, Numerical Optimization, Springer, Heidelberg; 2006.

[25] P. Hahnfeldt, D. Panigrahy, J. Folkman and L. Hlatky, "Tumor development under angiogenic signaling: A dynamical theory of tumor growth, treatment response, and postvascular dormancy", Cancer research, vol. 59, pp. 4770-4775, 1999.

[26] D. A. Drexler, L. Kovács, J. Sápi, I. Harmati and Z. Benyó, "Model-based analysis and synthesis of tumor growth under angiogenic inhibition: a case study", in Proc. IFAC WC 2011 18th World Congress of the International Federation of Automatic Control, Milano, Italy, pp. 3753-3758, 2011.

[27] D. A. Drexler, J. Sápi, A. Szeles, I. Harmati, A. Kovács and L. Kovács, "Flat control of tumor growth with angiogenic inhibition", in Proc. SACI 2012 - 6th International Symposium on Applied Computational Intelligence and Informatics (IEEE), Timisoara, Romania, pp. 179-183, 2012.

[28] J. Sápi, D. A. Drexler, I. Harmati, Z. Sápi and L. Kovács, "Linear state feedback control synthesis of tumor growth control in antiangiogenic therapy", in Proc. SAMI 2012 - 10th International Symposium on Applied Machine Intelligence and Informatics (IEEE), Herlany, Slovakia, pp. 143-148, 2012.

[29] A. Szeles, J. Sápi, D. A. Drexler, I. Harmati, Z. Sápi and L. Kovács, "Model-based angiogenic inhibition of tumor growth using modern robust control method", in Proc. IFAC BMS 2012 8th IFAC Symposium on Biological and Medical Systems, Budapest, Hungary, pp. 113-118, 2012.

[30] H. W. van Laarhoven, G. Gambarota, J. Lok. M. Lammens, Y. L. M. Kamm, T. Wagener, C. J. A. Punt, A. J. van der Klogel and A. Heerschap, "Carbogen breathing differentially enhances blood plasma volume and 5-fluorouracil uptake in two murine colon tumor models with a distinct vascular structure", Neoplasia., vol. 8, no. 6, pp. 477-487, 2006.

[31] Abcam, "B16 (mouse melanoma cell line) nuclear lysate," http://www. abcam.com/B16-Mouse-melanoma-cell-line-NuclearLysate-ab14638.html, 11.03.2013.

[32] W. W. Overwijk and N. P. Restifo, "B16 as a mouse model for human melanoma", Curr Protoc Immunol, vol. Chapter 20, p. Unit 20, 2001.

[33] E. D. Yorke, Z. Fuks, L. Norton, W. Whitmore, and C. C. Ling, "Modeling the development of metastases from primary and locally recurrent tumors: comparison with a clinical data base for prostatic cancer", Cancer Res., vol. 53(13), pp. 2987-2993, 1993.

[34] K. Flegal and C. Ogden, "Childhood Obesity: Are We All Speaking the Same Language?", Adv Nutr, vol. 2, no. 2, pp. 159S66S, 2011.

[35] J Kálmán, "Results of National Longitudinal Child Growth Survey from birth to 18 years of old I" (in Hungarian), $K S H$, vol. 83, 2006.
[36] CDC NCHS: 2000 CDC Growth Charts for the United States: Methods and Development. Vital and Health Statistics, Series 11, Number 246, 2002.

[37] J. Reiczigel, A. Harnos and N. Solymosi, Biostatistics not for statisticians (in Hungarian), Pars Kft, Budapest, 2010.

[38] J. Shaffer, "Multiple Hypothesis Testing", Ann Rev of Psych, vol. 46, pp. 561-584, 1995.

[39] R. Miller, Simultaneous Statistical Inference, Springer, New York, 1981.

[40] D. Scott, Multivariate density estimation: theory, practice, and visualization, Wiley, New York, 1992.

[41] P. Hall, J. S. Maron and B. Park, "Smoothed cross-validation", Probability Theory and Related Fields. vol. 92, pp. 1-20, 1992.

[42] E. Oda and R. Kawai, "Comparison between high-sensitivity Creactive protein (hs-CRP) and white blood cell count (WBC) as an inflammatory component of metabolic syndrome in Japanese", Internal Medicine, vol. 49, pp. 117-124, 2010.

[43] D. Gilbert-Diamond, A. Baylin, M. Mora-Plazas and E. Villamor, "Chronic inflammation is associated with overweight in Colombian school children", Nutr Metab Cardiovasc Dis, vol. 22, no. 3, pp. 244-251, 2012.

[44] A. Syrenicz, B. Garanty-Bogacka, M. Syrenicz, A. Gebala and M. Walczak "Low-grade systemic inflammation and the risk of type 2 diabetes in obese children and adolescents", Neuroendocrinology Letters, vol. 27, pp. 453-458, 2006.

[45] K. Ausk and G Ioannou, "Is obesity associated with anemia of chronic disease? A population-based study", Obesity, vol. 16, pp. 2356-2361, 2008.

[46] G. Lam and S. Mobarhan, "Central obesity and elevated liver enzymes”, Nutr Rev, vol. 62, pp. 394-399, 2004.

[47] P. Gholam, L Flancbaum, J. Machan, D. Charney and D. Kotler, "Nonalcoholic fatty liver disease in severely obese subjects", $\mathrm{Am} J$ Gastroenterol, vol. 102, pp.399-408, 2007. 